\title{
Biofeedback and Dance Performance: A Preliminary Investigation
}

\author{
Joshua Raymond, ${ }^{1}$ Imran Sajid, ${ }^{1}$ Lesley A. Parkinson, ${ }^{2}$ and John H. Gruzelier ${ }^{1,3}$
}

\begin{abstract}
Alpha-theta neurofeedback has been shown to produce professionally significant performance improvements in music students. The present study aimed to extend this work to a different performing art and compare alpha-theta neurofeedback with another form of biofeedback: heart rate variability (HRV) biofeedback. Twenty-four ballroom and Latin dancers were randomly allocated to three groups, one receiving neurofeedback, one HRV biofeedback and one no intervention. Dance was assessed before and after training. Performance improvements were found in the biofeedback groups but not in the control group. Neurofeedback and HRV biofeedback benefited performance in different ways. A replication with larger sample sizes is required.
\end{abstract}

KEY WORDS: alpha/theta neurofeedback; heart rate variability (HRV); dance.

\section{INTRODUCTION}

Clinical applications of neurofeedback have been extensively investigated, especially in the field of cognition (e.g., Egner \& Gruzelier, 2001; Vernon, Egner, Cooper, Compton, Neilands, Sheri, \& Gruzelier, 2003) and attention deficit hyperactivity disorder (Rossiter \& LaVaque, 1995; Fuchs, Birbaumer, Lutzenberger, Gruzelier, \& Kaiser, 2003; Vernon, Frick, \& Gruzelier, 2004). Alpha waves $(8-12 \mathrm{~Hz}$ ) are associated with relaxation (Kamiya, $1968)$ and theta $(4-7 \mathrm{~Hz})$ has been suggested partly to reflect a positive emotional state (Aftanas \& Golocheikine, 2001). Peniston and Kulkosky (1990) found that alpha/theta neurofeedback, when combined with other treatments, reduced rates of relapse in recovering alcoholics.

Less attention has been paid to the effects that neurofeedback training has on healthy subjects looking to alter their neural activity to enter a state of "peak performance." Egner and Gruzelier (2003) played soothing sounds to students at the Royal College of Music that represented the levels of their alpha and theta brainwaves, recording EEG from Pz. Other groups of students were given SMR and beta1 neurofeedback at $\mathrm{Cz}$, a sports psychology intervention, mental skills training or training in the Alexander Technique. There was also

\footnotetext{
${ }^{1}$ Imperial College London, St Dunstan's Road, London W6 8RF, UK.

${ }^{2}$ Brainhealth Ltd., c/o The Diagnostic Clinic, 50 New Cavendish Street, London W1G 8TL, UK.

${ }^{3}$ Address all correspondence to John Gruzelier, Division of Neuroscience \& Psychological Medicine, Imperial College London, St Dunstan's Road, London W6 8RP, UK; e-mail: j.gruzelier@imperial.ac.uk.
} 
a no-treatment control group. Students were videotaped performing a piece both before and after feedback training. These videos were then sent to expert assessors who were blind as to the time (pre- or post-training) and training group of the musician. The videos were marked on standard conservatory grading scales. It was found that participants in the alpha/theta feedback group improved their musical performance by up to two grade points (e.g., from a lower second to a first class grade), with no significant changes seen in the other groups. Artistry in performance was most affected. It could be expected in the light of theories of arousal in sport (Yerkes \& Dodson, 1908; Fazey \& Hardy, 1988) that the improvements were mediated by a reduction in pre-performance cognitive anxiety, and EEG changes were consistent with anxiety reduction (Egner \& Gruzelier, 2004a), but no difference in pre-performance anxiety, as measured by the Spielberger State Anxiety Inventory (STAI) was found between any of the groups, all of whom improved. Such was the interest generated by Egner and Gruzelier's results that an extension to other performing arts is worthwhile to examine what aspects of performance might be improved. The present study aimed to replicate Egner and Gruzelier's findings of musical performance enhancement following alpha/theta neurofeedback in a population of university dancers.

The present study also investigated heart rate variability (HRV) training, which is another form of biofeedback that is of potential interest to peak performance researchers. Research has associated increased HRV with emotional reactivity in infants (see Beauchaine, 2001), social competence in young children (Doussard-Roosevelt, Porges, Alemi, \& Scanlon, 1997), lowered aggression in adolescents (Mezzacappa et al., 1997) and lowered hostility in young adults (Sloan et al., 1994). Decreased HRV has been associated with both panic disorder and depression (Yeragani et al., 1993; Yeragani et al., 1991). Breathing six times a minute occurs in Catholic rosary prayer and Yogic mantras (Bernardi et al., 2001) and produces dramatic changes in HRV and baroreflex sensitivity. These changes in the balance of the autonomic nervous system may be instrumental in the pleasant feelings associated with prayer and yoga.

Turning to HRV training as an intervention, it seems clear that high HRV, especially at the same frequency as the baroreflex, has physiological and possibly psychological benefits. Gevirtz has investigated HRV biofeedback for rehabilitation following myocardial infarction as well as the treatment of panic disorder (Del Pozo \& Gevirtz, 2002; Berger \& Gevirtz, 2001), and Bessel and Gevirtz (1998) found breathing retraining reduced anxiety and improved performance in female gymnasts.

The present study therefore compared alpha/theta neurofeedback training and HRV training with a no-treatment control group in a population of dancers. It was hypothesised that both of these interventions would improve dancers' performance in different ways, because of their effects on the central and peripheral nervous systems. The present study was conducted as a preliminary step in constructing a programme of psychophysiological intervention to optimise performance in sportspeople and performing artists.

\section{METHOD}

\section{Participants}

Participants were 24 students from the Imperial College Dance Sport Team (mean age $=21.6$ years). Dancers performed in male-female pairs, and each pair was randomly 
allocated, matching for age and sex, to one of three groups. Group 1 received a/t training, group 2 received HRV training and group 3 received no training.

Due to seasonal absences and interruptions eight participants dropped out of the study, leaving six subjects receiving a/t, four receiving HRV and eight acting as controls. Due to the timing of university holidays, participants practised different amounts during the course of the study, and this is taken into account when calculating performance changes.

No participant had any history of physical or mental health problems, nor any experience or knowledge of biofeedback training. All participants were briefed before the study and gave their full written consent.

\section{Procedure-Alpha/Theta Neurofeedback}

Ten sessions of neurofeedback per participant were carried out over the course of four weeks using the Brainmaster (Ohio, USA) with the active electrode placed at $\mathrm{Pz}$ (following Egner \& Gruzelier, 2003; Egner \& Gruzelier, 2004a, 2004b who obtained significant results with 10 sessions of feedback) and referenced to the ear. Skin was prepared with NuPrep and electrodes attached with Ten 20 conductive paste. Impedances were checked by visual inspection of the raw signal.

Participants were seated in a comfortable chair in a darkened room and instructed to relax. Before the first session, participants were explained the underlying principles of neurofeedback and instructed to close their eyes and relax as deeply as possible, without falling asleep. All feedback was carried out with eyes closed.

A 2-minute, eyes-closed, feedback-free run was carried out to assess the individual's dominant alpha frequency (IAF), as recommended by Doppelmayr, Klimesch, Pachinger, and Ripper (1998). The modal frequency within the $8-12 \mathrm{~Hz}$ band was extracted from this initial run and an alpha band calculated as $1.5 \mathrm{~Hz}$ either side of this value. Theta was defined as a $3 \mathrm{~Hz}$ band whose median frequency lay $4 \mathrm{~Hz}$ below the IAF. For example, if a participant had an IAF of $10 \mathrm{~Hz}$, alpha was defined as $8.5-11.5 \mathrm{~Hz}$ and theta as $4.5-7.7 \mathrm{~Hz}$.

Feedback began after this initial assessment and took the form of sounds presented to participants through headphones. When participants' alpha power was higher than their theta power, a "babbling brook" sound was heard, and when theta was higher than alpha, this changed to "crashing waves." Each band also had an amplitude threshold, and suprathreshold bursts of alpha or theta were rewarded by a high- or low-pitched gong sound respectively. These thresholds were set manually by the experimenter and updated such that alpha and theta amplitudes were over these thresholds approximately $60 \%$ of the time.

Before each session of neurofeedback, participants were read a short induction that instructed them to focus on their breathing and the feedback sounds. They were told to imagine moving down a stream (for the babbling brook sound) and out towards the sea (for the crashing waves sound). They were told that, when they could hear the waves, they should visualise themselves dancing in the way they most wanted to dance. This instruction was based on the idea that guided imagery in a state of deep relaxation might help performance (e.g., Budzynski, 1977).

Each session lasted for 20 minutes, with EEG data being gathered in 20 one-minute "runs." The feedback sounds would then be faded out and the participant brought gently to full wakefulness. Participants were constantly monitored by the experimenter for excessive 
delta activity or sleep like behaviour, whereupon they would be tapped gently on the knee until they acknowledged that they were awake.

\section{Procedure-HRV Biofeedback}

HRV training was carried out using the Freeze-Framer (Boulder Creek, California, USA). This features a photoplethysmograph that attaches to a finger and software that presents the pulse pressure wave on a screen and plots a graph of heart rate over time. The variability of the heart rate curve is converted to a "score", which represents rhythmic changes in heart rate with breathing.

A 10-session protocol similar to that designed by Lehrer, Vaschillo, and Vaschillo (2000) was used for training. Using 10 sessions had the added advantage of controlling for therapist contact effects between the two biofeedback conditions. The goal of the training was to have participants breathe at their resonant frequency. The protocol was semiflexible and could be modified to accommodate different rates of learning. Participants were monitored throughout for signs of hyperventilation and instructed to breathe slowly but no more deeply than usual and to report any symptoms to the experimenter. Each session was, like the neurofeedback sessions, 20 minutes long.

\section{Procedure-Controls}

Control participants were informed of the nature of the study and that they would be receiving no intervention. They were instructed to carry on practising dance as normal.

\section{Dance Assessment}

Assessments of dance performance were carried out pre- and post-training. Dancers were rated on customised evaluation scales created by two qualified dance assessors with marks being awarded on a scale of one to five for technicality, musicality, timing, partnering skill, performing flair and overall execution. This is the standard scale used for National dance assessments. Whilst participants performed in pairs, they were evaluated individually. Two judges were used and their scores averaged. Judges were blind to participant group. One judge had been runner-up in the World Ballroom Championships.

\section{RESULTS}

Participants received on average $9 \pm 1.26$ sessions of biofeedback.

\section{Dance Performance}

Ratings for the two judges were averaged for each dancer across all subscales and are shown in Table I with standard deviations in brackets. As can be seen, all groups improved with practice. 
Table I. Mean and Standard Deviations of Age for the Three Groups

\begin{tabular}{lc}
\hline \multicolumn{1}{c}{ Group } & Mean age $(S D)$ \\
\hline Neurofeedback & $20.73(2.42)$ \\
HRV & $19.84(2.21)$ \\
Control & $21.05(2.51)$ \\
\hline
\end{tabular}

For the purposes of analysis, data were treated as nonparametric, due to the small number of subjects, the ordinal nature of the rating scales and the small size of the integers involved in scoring (1-5).

Pre-training scores were subtracted from post-training scores and the resultant difference scores were used for analysis. Most dancers went home for university holidays and so had six sessions of practice, but one dancer in the HRV group and three in the control group remained at university to train for upcoming competitions and had 12 sessions of practice. In the light of this, difference scores were divided by number of practice sessions for each participant to get an "improvement per practice session" score. These scores were then averaged to get a "practice-corrected average difference" score for the group as a whole. The difference scores, amount of practice had by each group and the practice-corrected difference scores are shown in Table II.

Mann-Whitney $U$ tests on the practice-corrected average difference scores showed that the neurofeedback group improved significantly $(z=2.452, p<0.05)$ more than the control group and that the HRV group also improved significantly $(z=2.389, p<0.05)$ more than the control group (see Fig. 1). There was no significant difference between the biofeedback groups $(z=0.176, \mathrm{~ns})$.

Neurofeedback and HRV training influenced the subscales of dance performance in different ways (see Fig. 2). Firstly, in support of the advantage to biofeedback shown by the averaged ratings, a Mann-Whitney $U$ test showed that biofeedback per se advantaged "Overall Execution" ( $z=2.080, p<0.05)$, whereas there were no advantages to the biofeedback groups for "musicality" $(z=0.897, p=0.41)$, "partnering" $(z=0.273, p=$ 0.83 ) or "performance flair" $(z=1.48, p=0.14)$.

However, there were two differentiated biofeedback effects. For the timing subscale, a Mann-Whitney $\mathrm{U}$ test showed the neurofeedback group to improve significantly more than controls $(z=2.688, p<0.05$ ), while for the technique subscale, the HRV group improved significantly more than controls $(z=2.138, p=0.05)$.

\section{Indices of Feedback Learning}

For neurofeedback, two indices of learning were calculated, a within-session learning coefficient (the correlation between theta/alpha ratio per minute block and block number

Table II. Mean and Standard Deviations of Pre- and Post Training Scores for the Three Groups

\begin{tabular}{lcc}
\hline \multicolumn{1}{c}{ Group } & Pre-training score & Post-training score \\
\hline Neurofeedback $(n=6)$ & $3.85(0.51)$ & $4.32(0.40)$ \\
HRV feedback $(n=4)$ & $3.44(0.40)$ & $3.96(0.32)$ \\
Control $(n=8)$ & $3.54(0.43)$ & $3.84(0.38)$ \\
\hline
\end{tabular}


Improvements in dancing performance with training

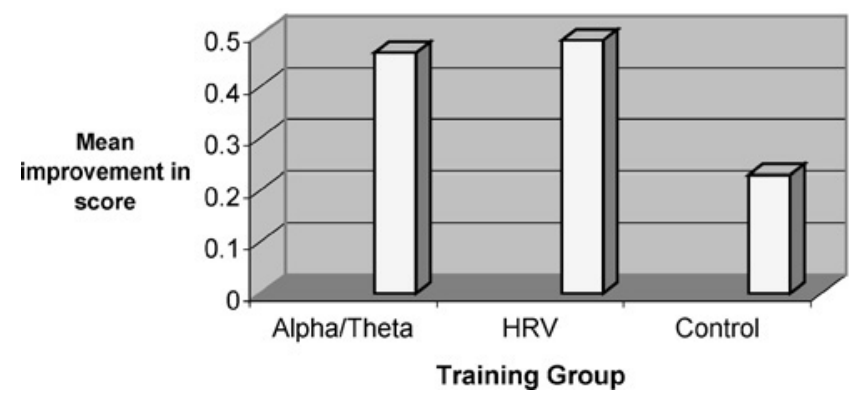

Fig. 1. Both the alpha/theta neurofeedback group and the HRV training group improved their dancing significantly more than the control group.

within a session) (mean $r=0.39$, range: $r=-0.04$ to 0.73 ) and an across-session coefficient (the correlation between the within-session coefficient and session number to establish whether within-session changes in theta/alpha ratios occurred more quickly across sessions) (mean $r=0.28$, range: $r=-0.05$ to 0.53 ) A feedback-learning coefficient was calculated for HRV training from the "entrainment" scores provided by the Freeze-Framer program (mean $r=0.20$, range: $r=-0.05$ to 0.78 ). None of the learning indices calculated correlated at $p=0.05$ with performance improvements. As can be seen in Table III the findings cannot be attributed to practice for the control group practised more.

\section{DISCUSSION}

The present study provides preliminary evidence that both alpha/theta neurofeedback and HRV biofeedback improve dance performance, as rated by judges blind to condition,

\section{Dance Imrovements by Subscale}

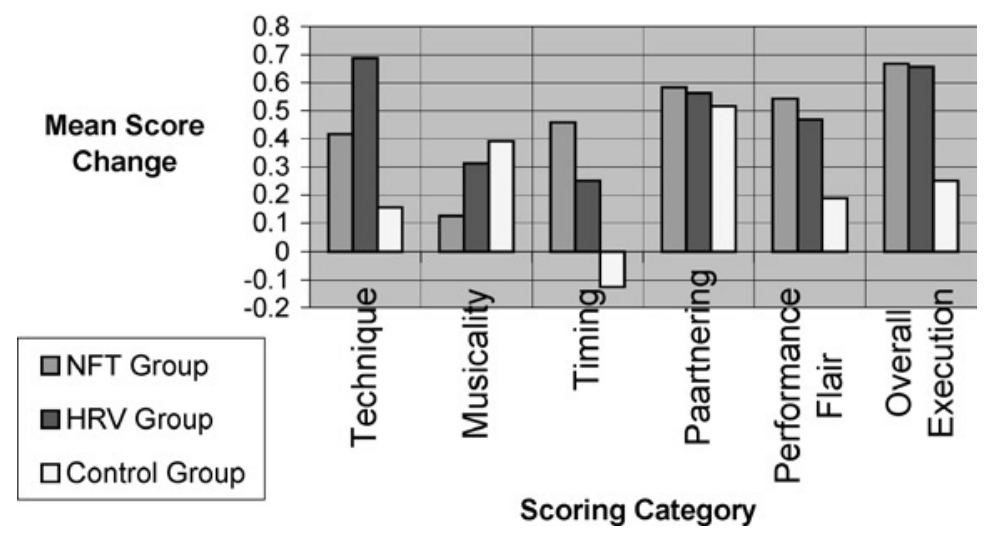

Fig. 2. Technique was significantly improved by HRV training. Timing was significantly improved by neurofeedback training. Overall execution was significantly improved by biofeedback. 
Table III. Pre-/Post-Training Difference Scores, Mean Number of Practice Sessions Per Person and Practice-Corrected Difference Scores for the Three Groups

\begin{tabular}{lccc}
\hline \multicolumn{1}{c}{ Group } & $\begin{array}{c}\text { Difference } \\
\text { score }\end{array}$ & $\begin{array}{c}\text { Mean number of } \\
\text { practice sessions }\end{array}$ & $\begin{array}{c}\text { Practice-corrected } \\
\text { difference score }\end{array}$ \\
\hline Neurofeedback & $0.47(0.43)$ & 6 & $0.47(0.43)$ \\
HRV feedback & $0.52(0.38)$ & 7.5 & $0.49(0.39)$ \\
Control & $0.30(0.42)$ & 8.25 & $0.23(0.40)$ \\
\hline
\end{tabular}

when compared to a no-treatment control group. This was seen in the average rating across subscales as well as in the "overall execution" summary scale. The two biofeedback approaches also disclosed some differentiated influences. The subscale "timing" was improved by neurofeedback, and the subscale "technique" was improved by HRV biofeedback. The dance assessors commented that, given the relatively long time course of dance improvement, even a half-point change is professionally significant. This extends the work of Egner and Gruzelier (2003) who found professionally significant improvements in artistry in conservatoire musicians in two segmented studies as a result of alpha/theta training.

Porges (2003) argued that HRV was associated with social gesture and expression because the "smart" vagus nerve that provides for RSA evolved at the same time as social gesture and facial expression. Dance has its origin in social rituals and communications and it is not unreasonable to suppose that high HRV, associated as it is with emotional reactivity and lowered aggression, could facilitate dancing.

The indices of neurofeedback learning showed no significant correlation with performance change. This was not in line with Egner and Gruzelier's findings of strong correlations between neurofeedback learning and performance change in musicians. One reason for the discrepancy may be that five out of six neurofeedback participants in the present study had theta higher than alpha at baseline, whereas this was true for only about $10 \%$ of Egner and Gruzelier's participants. A body of research in sports psychology suggests that relaxation and imagery are effective in enhancing performance (Wrisberg \& Anshel, 1989; Weinberg, Seabourne, \& Jackson, 1981), and it is plausible that the deeply relaxed theta over alpha state could facilitate performance-enhancing imagery. Participants in the present study did, on average, increase their theta/alpha ratios within sessions (and a review currently underway in our laboratory of EEG data from 38 individuals' shows within session profiles are broadly similar regardless of baseline alpha and theta levels) but it is possible that having theta over alpha all the time, as five out of six participants did, meant that the relationship between theta/alpha ratio and performance might have become less distinct. It is possible that these participants, unlike Egner and Gruzelier's, would have improved their performance by using guided imagery without feedback as they were in the theta over alpha state at baseline. Further investigation into the EEG dynamics of individuals whose theta is over alpha at baseline is required.

The present study has several limitations, most notably the small group sizes, and should therefore be considered exploratory. It could also be argued that, in comparing two intervention conditions to a no-intervention control group, the study is left vulnerable to therapist contact effects; the results observed could be merely due to the participants' having had any sort of contact with the experimenter at all. It seems unlikely that this is the entire explanation as the two interventions affected dance performance in different 
ways, something unlikely to result from a simple "placebo" brought about by experimenter contact and the belief that one was receiving an effective intervention. Nevertheless, a replication should be carried out with larger sample sizes. A mock-neurofeedback group instead of a no-treatment control and a pre-performance anxiety measure would also be informative.

The study, especially when considered alongside the findings of Egner and Gruzelier (2003), suggests that, pending further investigation with larger groups, neurofeedback and HRV training could be used as an adjunct to more traditional sports psychology interventions such as guided imagery. It adds to the growing body of research validating the efficacy of biofeedback for performance enhancement (Gruzelier \& Egner, 2005).

\section{ACKNOWLEDGMENTS}

The authors would like to thank Brainhealth for their generous sponsorship of this research.

\section{REFERENCES}

Aftanas, L. I., \& Golocheikine, S. A. (2001). Human anterior and frontal midline theta and lower alpha reflect emotionally positive state and internalised attention: High-resolution EEG investigation of meditation. Neuroscience Letters, 310, 57-60.

Beauchaine, T. (2001). Vagal tone, development, and Gray's motivational theory: Toward an integrated model of autonomic nervous system functioning in psychopathology. Developmental Psychopathology, 13(2), 183214.

Berger, B. C., \& Gevirtz, R. (2001). The treatment of panic disorder. A comparison between breathing retraining and cognitive behaviour therapy. Applied Psychophysiology and Biofeedback, 26(3), 227-228.

Bernardi, L., Sleight, P., Bandinelli, G., Cencetti, S., Fattorini, L., Wdowczyc-Szulc, J., \& Lagi, A. (2001). Effect of rosary prayer and yogic mantras on autonomic cardiovascular rhythms: Comparative study. British Medical Journal, 323, 1446-1449.

Bessel, J., \& Gevirtz, R. (1998). Effects of breathing retraining versus cognitive techniques on cognitive and somatic components of state anxiety and on performance of female gymnasts. Biological Psychology, 48(1), 18.

Budzynski, T. H. (1977). Tuning in on the Twilight Zone. Psychology Today, August.

Del Pozo, J., \& Gevirtz, R. (2002). The effect of resonant frequency cardiac biofeedback training on heart rate variability in a cardiac rehabilitation population. Applied Psychophysiology and Biofeedback, 27(4), 311.

Doppelmayr, M., Klimesch, W., Pachinger, T., \& Ripper, B. (1998). Individual differences in brain dynamics: Important implications for the calculation of event-related band power. Biological Cybernetics, 79, (1) 49-57.

Doussard-Roosevelt, J. A., Porges, S. W., Scanlon, J. W., Alemi, B., \& Scanlon, K. B. (1997). Vagal regulation of heart rate in the prediction of developmental outcome for very low birth weight preterm infants. Child Development, 68(2), 173-186.

Egner, T., \& Gruzelier, J. H. (2001). Learned self-regulation of EEG frequency components affects attention and event-related brain potentials in humans. Neuroreport, 12(18), 4155-4159.

Egner, T., \& Gruzelier, J. H. (2003). Ecological validity of neurofeedback: Modulation of slow-wave EEG enhances musical performance. Neuroreport, 14(9), 1221-1224.

Egner, T., \& Gruzelier, J. H. (2004a). The temporal dynamics of electroencephalographic responses to alpha/theta neurofeedback training in healthy subjects. Journal of Neurotherapy, 8(1), 43-58.

Egner, T., \& Gruzelier, J. H. (2004b). EEG biofeedback of low beta band components: Frequency-specific effects on variables of attention and event-related brain potentials. Clinical Neurophysiology, 115, 131-139.

Fazey, J., \& Hardy, L. (1988). The inverted-U hypothesis - a catastrophe for sport psychology. British Association of Sports Sciences Monograph, 1, Leeds, UK: National Coaching Foundation.

Fuchs, T., Birbaumer, N., Lutzenberger, W., Gruzelier, J. H., \& Kaiser, J. (2003). Neurofeedback treatment for attention-deficit/hyperactivity disorder in children: A comparison with methylphenidate. Applied Psychophysiology and Biofeedback, 28(1), 1-12.

Gruzelier, J. H., \& Egner, T. (2004) Physiological self-regulation: Biofeedback and neurofeedback. In A. Williamon (Ed.), Musical excellence; strategies and techniques to enhance performance (pp. 197-219). Oxford: Oxford University Press. 
Gruzelier, J., \& Enger, T. (2005). Critical validation studies of neurofeedback. Child and Adolescent Psychiatric Clinics of North America, 14, 83-104.

Kamiya, J. (1968). Conscious control of brainwaves. Psychology Today, 1, 56-60.

Lehrer, P. M., Vaschillo, E., \& Vaschillo, B. (2000). Resonant frequency biofeedback training to increase cardiac variability: Rationale and manual for training. Applied Psychophysiology and Biofeedback, 25(3), 177-191.

Mezzacappa, E., Tremblay, R. E., Kindlon, D., Saul, J. P., Arseneault, L., Seguin, J., Pihl, R. O., \& Earls, F. (1997). Anxiety, antisocial behavior and heart rate regulation in adolescent males. Journal of Child Psychology and Psychiatry and Allied Disciplines, 38(4), 457-469.

Peniston, E. G., \& Kulkosky, P. J. (1990). Alcoholic personality and alpha-theta brainwave training. Medical Psychotherapy, 3, 37-55.

Porges, S. W. (2003) The Polyvagal Theory: Phylogenetic contributions to social behavior. Physiology and Behaviour, 79(3), 503-513.

Raymond, J., Varney, C., Parkinson, L. A., \& Gruzelier, J. H. (2005). The effects of alpha/theta neurofeedback on personality and mood. Cognitive Brain Research (in press).

Rossiter, T. R., \& LaVaque, T. J. (1995). A comparison of EEG biofeedback and psychostimulants in treating attention deficit hyperactivity disorders. Journal of Neurotherapy, 1, 48-59.

Sloan, R. P., Shapiro, P. A., Bigger, J. T., Bagiella, E., Steinman, R. C., \& Gorman, J. M. (1994). Cardiac autonomic control and hostility in healthy subjects. American Journal of Cardiology, 74(3), 298-300.

Vernon, D., Frick, A., \& Gruzelier, J. H. (2004). Neurofeedback as a treatment for ADHD: A methodological review with implications for future research. Journal of Neurotherapy, 8, 53-82.

Vernon, D., Egner, T., Cooper, N., Compton, T., Neilands, C., Sheri, A., \& Gruzelier, J. H. (2003). The effect of training distinct neurofeedback protocols on aspects of cognitive performance. International Journal of Psychophysiology, 47, 75-85.

Weinberg, R. S., Seabourne, T. G., \& Jackson, A. (1981). Effects of visuo-motor behavior rehearsal, relaxation, and imagery on karate performance. Journal of Sport Psychology, 3, 228-238.

Wrisberg, C. A., \& Anshel, M. H. (1989). The effect of cognitive strategies on the free throw shooting performance of young athletes. The Sport Psychologist, 3, 95-104.

Yeragani, V. K., Pohl, R., Balon, R., Ramesh, C., Glitz, D., Jung, I., \& Sherwood, P. (1991). Heart rate variability in patients with major depression. Psychiatry Research, 37I(1), 35-46.

Yeragani, V. K., Pohl, R., Berger, R., Balon, R., Ramesh, C., Glitz, D., Srinivasan, K., \& Weinberg, P. (1993). Psychiatry Research, 46(1), 89-103.

Yerkes, R. M., \& Dodson, J. D. (1908). The relation of strength of stimulus to the rapidity of habit formation. Journal of Comparative Neurology and Psychology, 18, 459-482. 